\title{
Recherche de staphylocoques pathogènes dans du lait en poudre
}

\author{
par \\ Dr SANTOS OVEJERO del AGUA \\ Professeur de Microbiologie Immunologie à la Faculté Vétérinaire \\ de León (Espagne) \\ et \\ Dr GUILLERMO SUAREZ FERNANDEZ \\ Professeur Agrégé de la Chaire
}

Il est évident que les intoxications alimentaires d'origine staphylococcique se produisent plus fréquemment à mesure que la variation du niveau de vie affecte les préparations culinaires, phénomène parfaitement défini et prouvé dans divers pays.

Ce type de maladie, de symptomatologie spectaculaire est, malgré sa basse létalité, une expérience désagréable à laquelle l'homme lui-même peut être soumis et, par conséquent, devient un procès de grand intérêt sanitaire et social.

Le rôle du lait en poudre comme agent transporteur de staphylocoques et leurs toxines fut exposé par Anderson et Stone [1] et Armijo et al. [2], et depuis lors on a diagnostiqué des foyers toxiques dus à ce type de germes et dont l'origine fut la consommation de lait en poudre dans divers pays.

Une caractéristique différentielle importante de l'intoxication par cet aliment, dans les foyers étudiés, est basée sur des populations d'enfants à l'âge scolaire, lesquelles furent grandement affectées.

En Espagne on a suivi un programme scolaire d'alimentation des enfants, stimulé par l'U.N.I.C.E.F., établi par le Ministère de l'Education et Science et administré par le Service scolaire de l'Alimentation du susdit Ministère et par l'Administration des Produits Laitiers. Ce Service emploie, dans ces buts, des milliers de tonnes de lait en poudre qui lui sont fournies par des industries nationales. 
Le manque de recherches, dans notre pays, sur le sujet de notre étude et la possibilité de ce que certaines perturbations de nature gastro-entérique dans des collectivités scolaires puissent être d'origine staphylococcique ont été les motifs qui nous ont conduit à effectuer ce travail.

\section{Echantillonnage et techniques d'étude}

On a étudié un total de 168 échantillons de lait en poudre provenant de différentes industries de transformation laitière, situées toutes dans notre pays. Les échantillons sont représentatifs de la production totale de lait en poudre en Espagne et on les a obtenus de différents lots commerciaux et d'industries productrices.

Nombre de colonies : Pour la détermination du nombre de colonies de staphylocoques on a suivi la méthode décrite par Harrigan et Mc Cance [3], ensemençant $1 \mathrm{~cm}^{3}$ de chaque dilution, faite à partir de lait en poudre en solution Ringer 1:4, dans des boîtes de Petri, en mélangeant, comme d'habitude, avec le milieu fondu et à la température de $45^{\circ} \mathrm{C}$.

Le flacon, à fermeture hermétique, avec le reste de la dilution de lait (10 g de lait en poudre et complétant le volume à $100 \mathrm{~cm}^{3}$ ) fut incubé, dans tous les cas, à $37^{\circ} \mathrm{C}$ pendant $48 \mathrm{~h}$, à côté des boîtes de Petri. Autrement dit, on employa la solution mère de lait en poudre elle-même, dans une proportion proche à celle employée pour l'alimentation humaine, comme un moyen d'enrichissement et afin de découvrir les échantillons qui ne croissaient pas sur le milieu solide distribué sur la boîte, à cause du petit nombre de staphylocoques qu'ils contenaient, ce qui signifiait leur absence dans $0,1 \mathrm{~g}$ de lait en poudre; dans ce cas, on employait le milieu liquide de lait en poudre dilué et incubé pour ensemencer, à partir de luimême, d'autres boîtes de Petri avec un milieu sélectif capable de démontrer, le cas échéant, la présence de staphylocoques.

Les colonies de staphylocoques de différents aspects sur un milieu de culture à base de chlorure de sodium comme agent sélectif, lactosé, avec de la fuchsine acide comme indicateur et potentiel d'oxyde réduction restreint [4] furent isolées et conservées pour leur postérieure étude.

Etude biochimique: Les souches isolées furent soumises aux essais suivants :

Réduction du tellurite de potassium [5].

Détermination de phosphatase [6].

Lipolyse dans du jaune d'œuf [7].

Production de désoxyribonucléase [8]. 
Fermentation anaérobique de mannitol [9].

Réaction de coagulase [10].

Etude des différents types d'entérotoxine : On employa la technique de micro-immuno-diffusion de Wadsworth [11] adaptée par Casman et al. [12] à l'étude et à la caractérisation des entérotoxines staphylococciques. Dans la production d'entérotoxine on employa le milieu proposé par Casman [13]. Les souches qui ne produisaient pas une croissance abondante à $\mathrm{pH} 5,5$ furent cultivées sur le même milieu à un $\mathrm{pH}$ de 6,8 .

Les sérums antitoxiques (correspondant aux types A, B, C et D de toxine) nous furent fournis par les Docteurs Casman (Department of Health, Education and Welfare, Washington) et Bergdoll (Food Research Institute, Madison, Wisconsin).

Croissance des staphylocoques coagulase positifs isolés dans du lait concentré : On vérifia le degré de croissance des souches de staphylocoques coagulase positive, isolés du lait en poudre, dans des laits concentrés de 35 p. 100,40 p. 100 et 45 p. 100 d'extrait sec total, après une préalable incubation à la température de $35^{\circ} \mathrm{C}$, $40^{\circ} \mathrm{C}, 45^{\circ} \mathrm{C}$ et $50^{\circ} \mathrm{C}$ pendant 2 et $4 \mathrm{~h}$.

Croissance du lait en poudre reconstitué : On chercha également la caractéristique de croissance dans du lait en poudre écrémé, reconstitué dans une proportion de $10 \mathrm{~g}$ de lait pour $100 \mathrm{~g}$ d'eau courante. Les températures de croissance essayées furent de $15^{\circ} \mathrm{C}$, $20^{\circ} \mathrm{C}, 25^{\circ} \mathrm{C}, 30^{\circ} \mathrm{C}$ et $35^{\circ} \mathrm{C}$.

Typification par des bactériophages : On a utilisé dans nos études, les phages de la Série Internationale, fournis par le "Central Public Health Laboratory ", Colindale (Londres), en suivant la technique recommandée par ce Centre.

\section{Résultats}

Nombre de colonies : Le chiffre le plus élevé a correspondu à l'échantillon $n^{\circ} 56$, avec 9400 staphylocoques par g. On n'isola aucun staphylocoque des 45 échantillons, et 30 contenaient moins de 10 staphylocoques par $\mathrm{g}$ (point en $0,1 \mathrm{~g}$ ).

Essais biochimiques : On isola 104 souches du genre Staphylococcus Rosenbach 1884, du total de 168 échantillons de lait en poudre étudiés. Des 104 souches, 24 seulement se révélèrent être coagugulase positive, ce qui signifie que 14 p. 100 des échantillons seulement contenaient des staphylocoques coagulase positive.

La totalité des souches coagulase positive montrèrent leur pouvoir de réduire le tellurite ainsi que 30 des 80 coagulase négative. 
Un total de 35 souches, dont 15 étaient coagulase positives et 20 négatives, résultèrent positives à l'essai de phosphatase.

La production de désoxyribonucléase put être observée dans un total de 42 souches, dont 16 seulement étaient coagulase positive.

Dans du milieu contenant du jaune d'œuf, 6 souches seulement, dont 5 étaient coagulase positive, montrèrent un halo d'opacité due à la lipolyse.

Un ensemble de 26 souches, dont 24 étaient coagulase positives et 2 négatives, furent cataloguées comme étant positives à la réaction du mannitol (fermentation anaérobique).

Types d'entérotoxine : Des 24 souches coagulase positive soumises à essai, seulement 6 ont montré une réaction positive correspondant à l'antisérum type $\mathrm{A}$, et l'une d'elles au type $\mathrm{D}$ aussi, après une préalable immersion pendant $15 \mathrm{mn}$ dans de l'acétate de cadmium à 1 p. 100 .

Croissance dans du lait concentré : On commença avec une inoculation d'environ 10000 staphylocoques par $\mathrm{cm}^{3}$. La population microbienne intégrante de la suspension standard inoculée fut obtenue en mélangeant des cultures incubées dans du bouillon de cerveau et de coeur de veau $(\mathrm{BHI})$ à la température de $35^{\circ} \mathrm{C}$ pendant $18 \mathrm{~h}$. Les souches utilisées dans les susdites cultures furent les 24 coagulase positives isolées du lait en poudre pendant le développement de ce travail.

Les valeurs et les caractéristiques de la croissance furent :

\section{TABLEAU 1}

Concentration initiale de germes : $10^{4}$ par $\mathrm{cm}^{3}$ de lait concentré Incubation : 2 et $4 \mathrm{~h}$

\begin{tabular}{|c|c|c|c|c|c|c|c|c|}
\hline \multirow{3}{*}{$\begin{array}{c}\begin{array}{c}\text { p. } 100 \\
\text { extrait sec }\end{array} \\
35\end{array}$} & \multicolumn{8}{|c|}{ Températures } \\
\hline & \multicolumn{2}{|c|}{$35^{\circ} \mathrm{C}$} & \multicolumn{2}{|c|}{$40^{\circ} \mathrm{C}$} & \multicolumn{2}{|c|}{$45^{\circ} \mathrm{C}$} & \multicolumn{2}{|c|}{$50^{\circ} \mathrm{C}$} \\
\hline & $2.10^{5}$ & $5.10^{7}$ & $10^{6}$ & $5.10^{6}$ & $10^{5}$ & $10^{6}$ & $10^{4}$ & $3.10^{2}$ \\
\hline 40 & $10^{5}$ & $10^{7}$ & $5.10^{5}$ & $2.10^{\mathrm{a}}$ & $5.10^{4}$ & $10^{6}$ & $5.10^{3}$ & $10^{2}$ \\
\hline 45 & $7.10^{4}$ & $9.10^{6}$ & $10^{5}$ & $10^{6}$ & $2.10^{4}$ & $6.10^{5}$ & $10^{3}$ & $8 \times 10$ \\
\hline
\end{tabular}

Croissance dans du lait en poudre reconstitué : La proportion de croissance dans les conditions de notre étude, en utilisant l'antérieur inoculum, fut la suivante : 
TABLEAU 2

Nombre initial de germes : $10^{4}$ par $\mathrm{cm}^{3}$ de lait reconstitué

Concentration de produit en extrait sec total : $10 \mathrm{~g} \mathrm{p} .100$

\begin{tabular}{c|r|r|r|r|r}
\hline \multirow{2}{*}{ Heures } & \multicolumn{5}{|c}{ Températures } \\
\cline { 2 - 6 } & $15^{\circ} \mathrm{C}$ & $20^{\circ} \mathrm{C}$ & $25^{\circ} \mathrm{C}$ & $30^{\circ} \mathrm{C}$ & $35^{\circ} \mathrm{C}$ \\
\cline { 2 - 6 } 2 & $2.10^{4}$ & $10^{5}$ & $3.10^{5}$ & $5.10^{5}$ & $8.10^{5}$ \\
4 & $8.10^{4}$ & $5.10^{5}$ & $10^{8}$ & $7.10^{8}$ & $10^{7}$ \\
8 & $10^{8}$ & $10^{7}$ & $4.10^{7}$ & $10^{7}$ & $5.10^{7}$ \\
16 & $2.10^{8}$ & $8.10^{8}$ & $10^{9}$ & $5.10^{9}$ & $9.10^{9}$ \\
24 & $10^{9}$ & $5.10^{9}$ & $8.10^{9}$ & $2.10^{10}$ & $5.10^{10}$ \\
48 & $2.10^{9}$ & $7.10^{9}$ & $1.10^{10}$ & $2.10^{10}$ & $4.10^{10}$ \\
\hline
\end{tabular}

Typification par phages : A côté des caractéristiques de lysotique qui semblent révéler une origine humaine, sensibles à plusieurs types parmi les suivants : $80 / 6 / 47 / 53 / 54 / 75 / 81$, il y a encore d'autres qui se présentent plus fréquemment et qui sont plus proches des souches de St. aureus d'origine animale, avec des tableaux de lyse dans lesquels on trouve surtout les types $42 \mathrm{E}$ et $42 \mathrm{D}$.

\section{Discussion}

Une fois démontrée, par Anderson et Stone [1], et Armijo et al. [2], la possibilité que le lait en poudre causait des intoxications chez l'homme dues à la présence d'entérotoxine staphylococcique dans le susdit produit, quelques auteurs indiquent que le lait concentré favorise la croissance des staphylocoques et, probablement, la production d'entérotoxine (Hobbs [14], Heineman [15], Crossley et Campling [16]).

$\mathrm{Vu}$ et considéré le problème d'après les connaissances actuelles sur les propriétés des différents types d'entérotoxine staphylococcique, ainsi que d'après le procès d'élaboration du lait en poudre, la plus grande probabilité de contamination est dans les points suivants :

a) Pasteurisation défectueuse du lait à concentrer, suivie d'une rétention excessive du lait concentré avant sa dessiccation.

b) Contamination dans le propre récipient ou tank de rétention.

c) Contamination du lait en poudre pendant l'ensachage.

Dans les cas a) et b) il n'est pas probable que des germes du genre Staphylococcus y survivent, puisque le lait concentré doit 
subir un chauffage avant sa pulvérisation, suffisant en tout cas pour détruire les susdits germes. Nous ne devons pas oublier non plus le propre effet de la dessiccation avec une température de l'air de $180^{\circ} \mathrm{C}$ à l'entrée dans la tour de dessiccation et d'environ $90^{\circ} \mathrm{C}$ à la sortie. Par contre, l'entérotoxine staphylococcique, en partie thermo-résistante, peut se trouver dans le produit final.

Dans le cas c) il ne peut pas exister de l'entérotoxine préformée dans l'aliment et l'unique danger dans ce cas est qu'en reconstituant le lait en poudre, celui-ci contienne des souches de staphylocoques entérotoxiques en quantité suffisante pour obtenir une croissance abondante dans le milieu liquide formé, quand on le maintient à une température optimale pour le développement microbien. Ce danger sera plus grand actuellement, dans la reconstitution massive dans des vaches mécaniques et dans des climats chauds.

D'accord avec ces idées, nous avons projeté notre travail en réalisant une ample étude sur le contenu en germes viables du genre Staphylococcus de différents lots commerciaux de lait en poudre, une étude biochimique et typification par phages, afin d'obtenir une caractérisation de la microflore staphylococcique de cette origine, l'étude des différents types d'entérotoxine des germes coagulase positive et les possibilités de croissance dans du lait concentré écrémé, avec les proportions d'extrait sec total appropriées par la dessiccation et aussi dans du lait en poudre reconstitué.

Dack [17] indiquait que dans les souches toxigéniques de staphylocoques, la production d'entérotoxine est fonction de la croissance et vu les difficultés techniques que, dans ce cas suppose l'essai objectif d'extraction, purification et titrage de l'entérotoxine elle-même, nous avons choisi la caractéristique de croissance microbienne comme un index de probabilité de formation de toxine.

D'après les résultats obtenus, nous pouvons affirmer que, même étant donné le petit pourcentage de souches entérotoxigéniques qui semblent se trouver dans la population étudiée et la petite proportion du nombre de staphylocoques par g, avec des maximums inférieurs à 10000 par g, et une moyenne de 370 , le plus grand danger est de maintenir le lait reconstitué à une température ambiante pendant plusieurs heures.

Seulement dans des cas de rétention du lait concentré avant sa dessiccation à des températures comprises entre $35^{\circ} \mathrm{C}$ et $45^{\circ} \mathrm{C}$, et pendant plus de $4 \mathrm{~h}$, ce qui n'est pas possible dans un procédé de fabrication normal, on pourrait dire que l'entérotoxine staphylococcique pourrait se trouver dans le produit final.

\section{Rés u m é}

On a fait une étude sur 168 échantillons de lait en poudre provenant d'industries nationales afin de préciser l'effet du problème des intoxications par l'entérotoxine staphylococcique de cette origine. 
Les déterminations réalisées dans le but proposé furent : énumération de colonies, caractérisation biochimique des souches isolées (essais de coagulase, phosphatase, désoxyribonucléase, fermentation du mannitol, lipolyse, réduction du tellurite), typification par des bactériophages, étude des différents types d'entérotoxine, croissance des souches de coagulase positives dans du lait en poudre concentré et reconstitué.

D'après les résultats obtenus, on déduit que, même en considérant le petit pourcentage de souches entérotoxigéniques qui semblent se trouver dans la population étudiée et la petite proportion du nombre de staphylocoques par g, avec une moyenne de 370 , le plus grand danger serait de maintenir le lait reconstitué à une température ambiante pendant plusieurs heures.

Dans un procédé normal de fabrication, avec une rétention de moins de $1 \mathrm{~h}$ du lait concentré avant sa dessiccation, il ne serait pas possible, à n'importe quelle température du lait retenu, de former de l'entérotoxine, laquelle se trouverait dans le produit final à cause de sa thermo-résistance.

\section{Su m m ary}

168 spray dried milk samples have been studied from Spanish dairies in order to establish the scope in the problem of food poisoning outbreaks due to staphylococcal enterotoxin of this origin.

The preformed tests to follow this line were: staphylococcal colony counts, biochemical characteristics of the isolated strains (coagulase, phosphatase, desoxyribonuclease, mannitol fermentation, lipolitic activity, potassium tellurite reduction) phage typing, detection of the types of enterotoxin, growth of the coagulase positive strains in evaporated milk and in reconstituted powder milk.

From the results obtained it has been deduced that despite the small percentage of enterotoxigenic strains among the studied microflora and the low proportion in the number of staphylococci per gram, with an average of 370 , it would be dangerous to maintain, at room temperature, for several hours, the reconstituted milk.

In a normal spray dried milk process, with a holding period of the concentrated milk before drying during less than one hour, it would not be possible, whichever the temperature might be, to cause enterotoxin to be produced, which avoids its presence in the final product.

\section{Bibliographie}

[1] ANderson (P. H. R.) y Stone (D. M.) (1955). - J. Hygiene, 53, 387.

[2] Armiso (R.), Henderson (D. A.), Timothee (R.) y Robinson (H. B.) (1957). Am. J. Public Health, 47, 1093.

[3] Harrigan (W. F.) y Mc CANCE (M.) (1968). - Métodos de laboratorio en microbiologia. Edit. Academia : Leôn (Espana). 
[4] Suarez (G.) (1966). - An. Fac. Vet. Leôn, 8, 141.

[5] Baird-Parker (A. C.) (1962). - J. Appl. Bact., 25, 12.

[6] Barber (M.) y KuPer (J. W. A.) (1951). - J. Path. Bact., 63, 65.

[7] Alder (V. G.) Guillespie (W. A.) y Herdan (G.) (1953). - J. Path. Bact., 64, 205.

[8] Weeckman (B.) y Catlin (B. W.) (1957). - J. Bact. 73, 747.

[9] Mossel (D. A.) (1962). - J. Bact., 84, 1140.

[10] Thatcher (F. S.) y CLARK (D. S.) (1968). - Microorganisms in foods. University of Toronto Press, Toronto (Canada).

[11] Wadsworth (C.) (1957). - Int. Arch. Allergy Appl. Immunol., 10, 355.

[12] Casman (E. P.), Bennett (R. W.), Dorsey (A. E.) y Stone (J. E.) (1969). Health Lab. Sci., 6, 185.

[13] Casman (E. P.) y Bennett (R. W.) (1963). - J. Bact., 86, 18.

[14] Новвs (B. C) (1955). - J. Appl. Bact., 18, 484.

[15] Heinemann (B.) (1957). - J. Dairy Sci., 40, 1585.

[16] Crossley y Campling (M.) (1957). - J. Appl. Bact., 20, 65.

[17] Dack (G. M.) (1962). - En Ayres (J.C.) et al. "Chemical and Biological. Hazards in Food ». Ames : Iowa State University Press.

Reçu pour publication en décembre 1970. 